\title{
Immunology of Recurrent Spontaneous Abortion
}

\section{ART ICLE INFO}

\section{Article Type}

Systematic Review

\section{Authors}

Roumandeh N. ${ }^{1} M S C$

Zare A.* PhD,

Saremi A. ${ }^{2} M D$
How to cite this article Roumandeh N, Zare A, Saremi A. Immunology of Recurrent Spontaneous Abortion. Sarem Journal of Reproductive Medicine. 2018; 2(3):121-126.
*Sarem Fertility \& Infertility Research Center (SAFIR), Sarem Women's Hospital, Tehran, Iran

${ }^{1}$ Sarem Cell Research Center (SCRC), Sarem Women's Hospital, Tehran, Iran

${ }^{2 " S a r e m ~ F e r t i l i t y ~ \& ~ I n f e r t i l i t y ~ R e-~}$ search Center (SAFIR)" and "Sarem Cell Research Center (SCRC)", Sarem Women's Hospital, Tehran, Iran

\section{Correspondence}

Address: Sarem Women's Hospital, Basij Square, Phase 3, Ekbatan Town, Tehran, Iran. Postal Code: 1396956111

Phone: +98 (21) 44670888

Fax: +98 (21) 44670432

ahadzr@gmail.com

\section{Article History}

Received: March 5, 2017

Accepted:June 25, 2017

ePublished: August 15, 2018

\section{A B S T R A C T}

Introduction Recurrent Spontaneous Abortion (RSA) is one of the most common complications in reproductive ages. Several factors such as genetic, anatomical, and endocrine disorders, infection, and environmental and immunological factors have been involved in RSA. In cases of abortions, whose reasons are known, the chance of success in pregnancy can be increased after the elimination of the defective factors; but, in women with a history RSA with an unknown reason, there is an imbalance in the regulatory mechanisms of immune cells, and even the pattern of immunological cells changes in these women. Actually, an inappropriate immune response is often associated with pregnancy loss. Immunologic factors involved in patients with RSA have gradually been identified, using diagnostic methods and several studies. The present study was conducted with the aim of reviewing RSA immunology and new findings in this field. In this study, more than 40 articles on immunologic factors involved in RSA were reviewed.

Conclusion Based on the current findings related to the immunology of pregnancy, due to the extensive role of the immunologic factors in this disorder, more accurate identification of the functional role of each immunologic factor becomes more important. Also, new therapeutic approaches will be addressed to the researchers in this field based on the changes in the function of immune system.

\section{Keywords Recurrent Spontaneous Abortion; Immunologic Factors; Immune System Phenomena}

\section{I T A T I O N L INKS}

[1] Recurrent miscarriage: Causes, evaluation and ... [2] The clinical use of karyotyping spontaneous ... [3] Future directions of failed implantation and recurrent miscarriage ... [4] An update in recurrent spontaneous ... [5] Immune etiology of recurrent pregnancy loss ... [6] Inflammation and the balance of Treg and Th17 cells in transplant rejection ... [7] Natural killer cells and regulatory $\mathrm{T}$ cells in early pregnancy ... [8] Reproductive ... [9] Immune profiling in patients with recurrent ... [10] Autoantibodies and prediction of reproductive ... [11] Autoimmunity and recurrent pregnancy ... [12] Mixed lymphocyte reaction blocking factors (MLR-Bf) as potential biomarker for indication and efficacy of paternal lymphocyte immunization in recurrent ... [13] Joining the immunological dots in recurrent ... [14] Changes and clinical significance of peripheral blood helper T lymphocyte and natural killer (NK) cells in unexplained ... [15] Characterization of the subsets of human NKT-like cells and the expression of Th1/Th2 cytokines in patients with unexplained recurrent ... [16] The Th1/Th2/Th17/Treg paradigm induced by ... [17] IL10-producing macrophages preferentially clear ... [18] The balance of the immune system between T cells and NK cells in ... [19] The deregulation of regulatory T cells on interleukin17-producing $\mathrm{T}$ helper cells in patients with unexplained early ... [20] Variations in T-helper 17 and Regulatory T Cells during ... [21] A physiological role for inducible ... [22] An imbalance in interleukin-17 ... [23] Intravenous immunoglobulin therapy affects $\mathrm{T}$ regulatory ... [24] Combined oral prednisolone and ... [25] Low-molecular-weight heparin ... [26] Low molecular weight heparin ... [27] Treatment options and pregnancy outcome in ... [28] Aspirin plus heparin or aspirin ... [29] Progesterone supplementation in women ... [30] The basis and value of currently used ... [31] Intravenous immunoglobulin (IVIG) and ... [32] The effects of immunotherapy ... [33] Diagnosis and treatment of recurrent miscarriage associated with immunologic disorders: Is paternal ... [34] Controlled trial of treatment of recurrent spontaneous ... [35] Lymphocyte immunotherapy and its probable ... [36] Human leukocyte antigen may predict outcome of primary ... [37] Possible mechanisms of immunotherapy for maintaining pregnancy in recurrent spontaneous ... 
جنين توسط سيستم ايمنى مادر رد مىشود. در سالهاى متمادى،

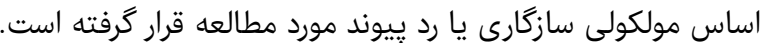

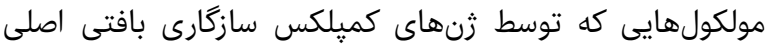

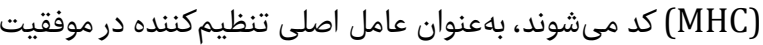

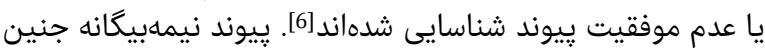

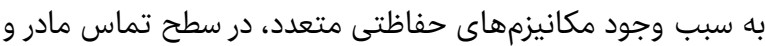
جنين طى باردارى حفظ مى شود. باردارى از ديدگًاه ايمونولوزيك بهدنبال ايجاد التهاب كاب كنترلشده

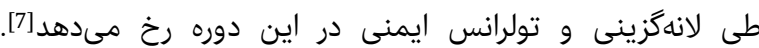

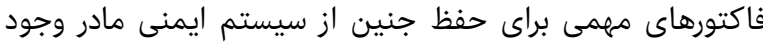

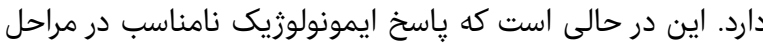

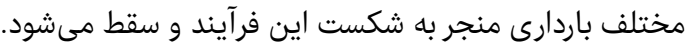

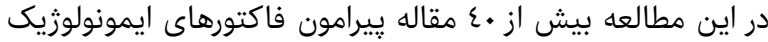

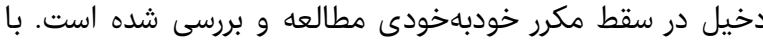

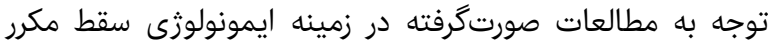
خودبهخودى، فاكتورهاى متعددى در اين عارضه دخيل إند كه ده در

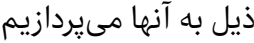

\section{فاكتورهاى اتوايميون}

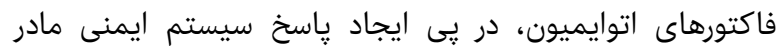

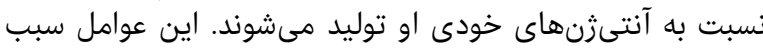

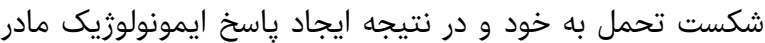

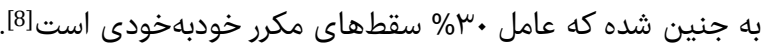

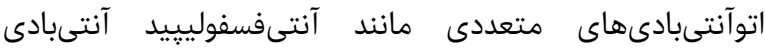

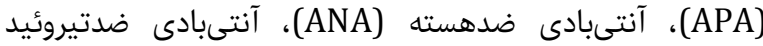

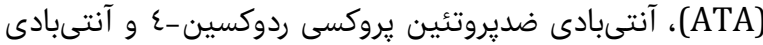

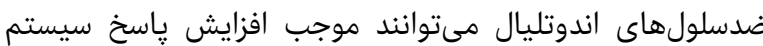

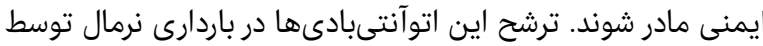

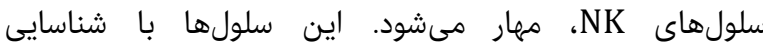

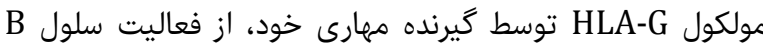

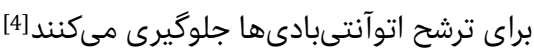

آنتىبادى آنتىفسفوليييد آنتيد (APA)

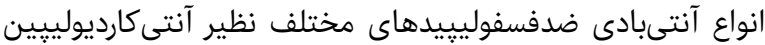

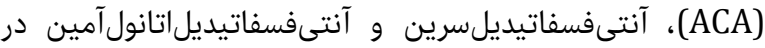

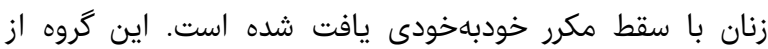

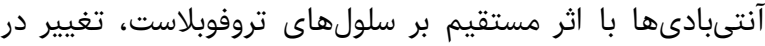

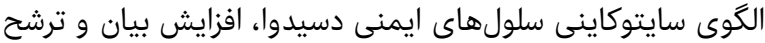

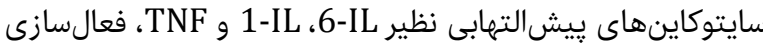

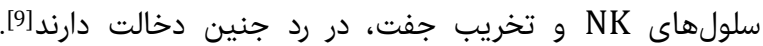

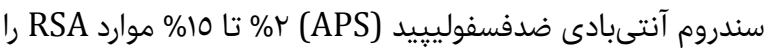

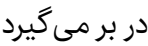

آنتىبادى ضدهسته (ANA)

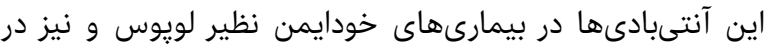

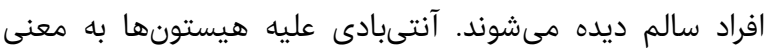

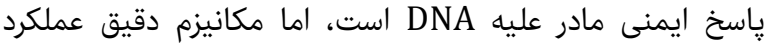
در رد جنين هنوز بلهدرستى شناسايى نشده استئ مادئ ميزان ANA

آنتىبادى ضدتيروئيد (ATA)

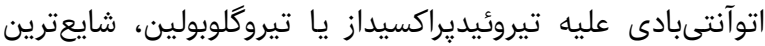

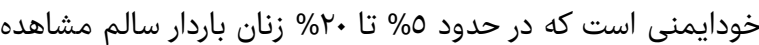

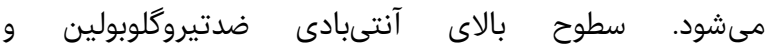

ايمونولوزى سقط مكرر خودبهخودى

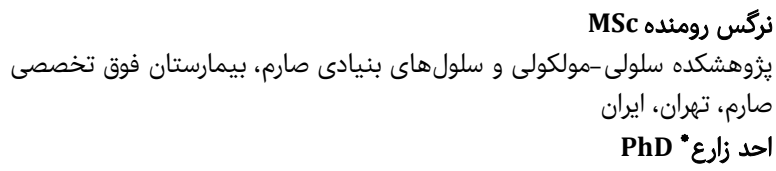

مركز تحقيقات بارورى و نابارورى صارم، بيمارستان فوق تخصصى صارم، تهران، ايران

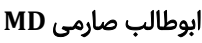

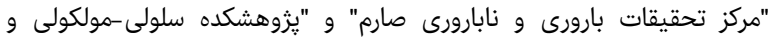
سلولهاى بنيادى صارم"، بيمارستان فوق تخصصى صاروى صارم، تهران، ايران "بران

جكيده

مقدمه: سقط مكرر خودبهخودى (RSA) از جمله مشكلات متداول در سنين

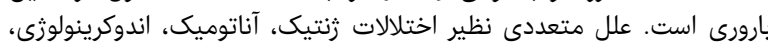

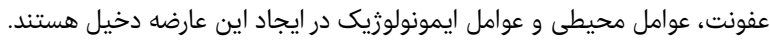

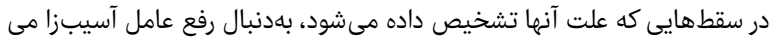

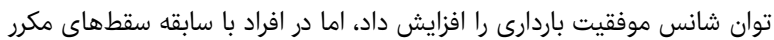

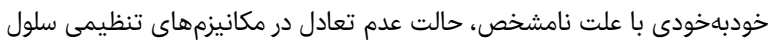

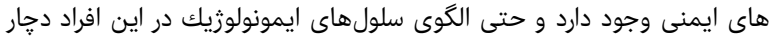

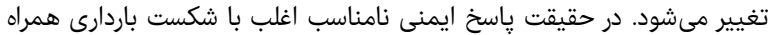

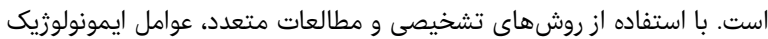

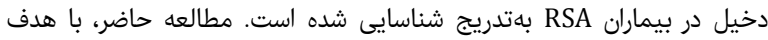

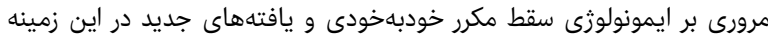

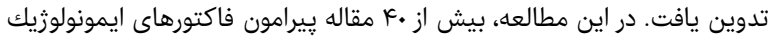

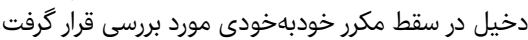

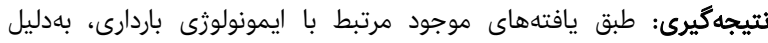

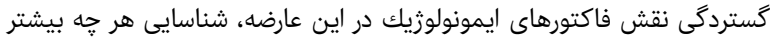

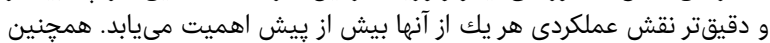

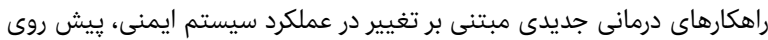

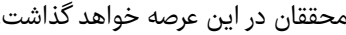
كليدوازهها: سقط مكرر خودبه خودى، عوامل ايمونولوزيك، ياسخ ايمنى

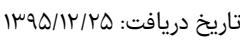

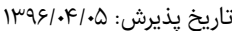

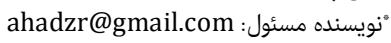

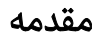

سقط مكرر خودبه خودى (RSA)، بهصورت سه يا بيش از سه سقط

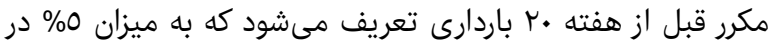

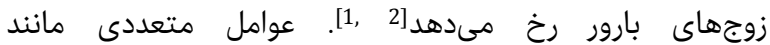

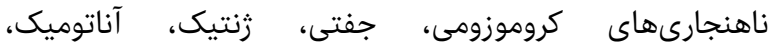

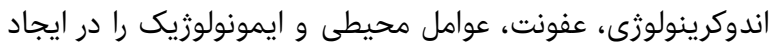

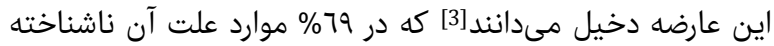

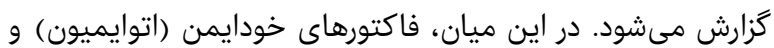

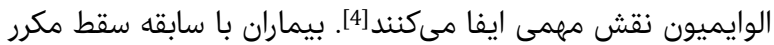

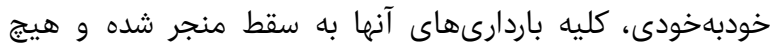

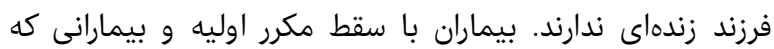

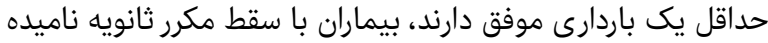

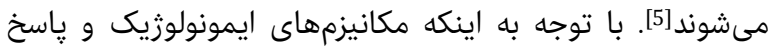

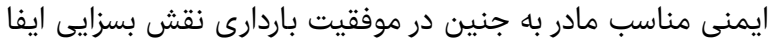

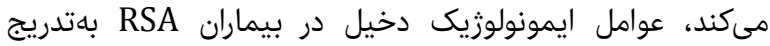

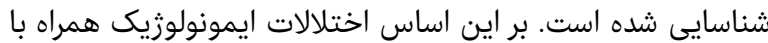

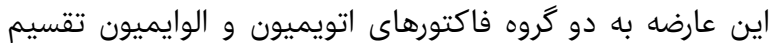

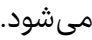
طى باردارى، جنين نيمهآلوزنيك از سوى سيستم ايمنى مادر مورد

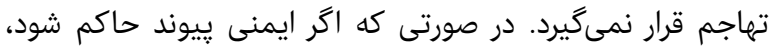


خون محيطى افزايش گرايش به سمت ايمنى نوع Th1 ران بشان

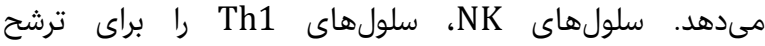

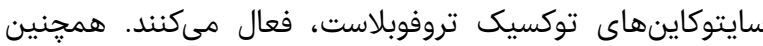

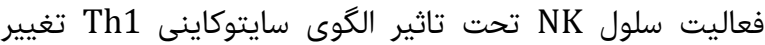

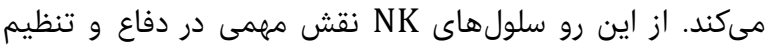

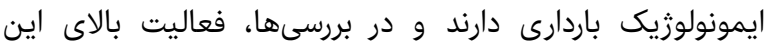

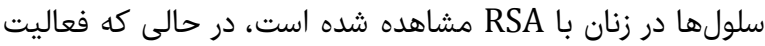

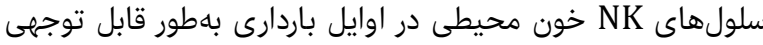

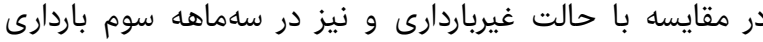

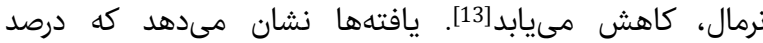

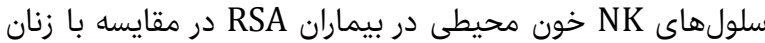

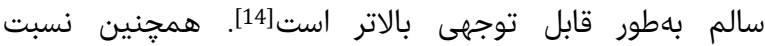
سلولهاى uNK/pNK در خون محيطى اين بيماران در مقايسه باليا

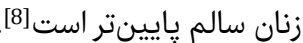

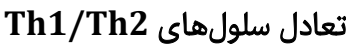

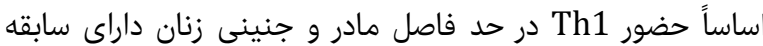

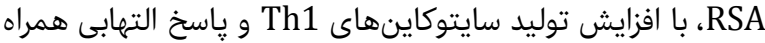

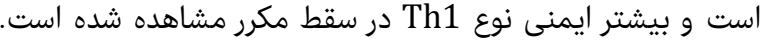

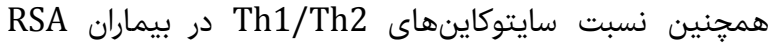

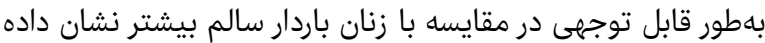

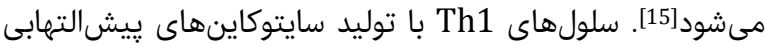

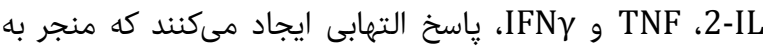
مرك سلولهاى تروفوبلاست مى شود. سطح بالاى

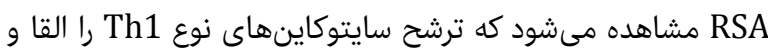

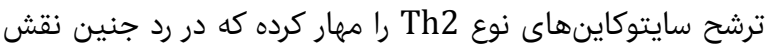

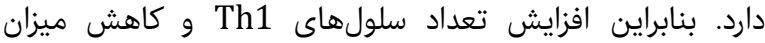

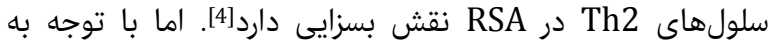

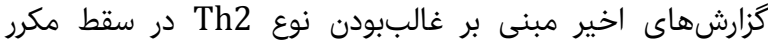

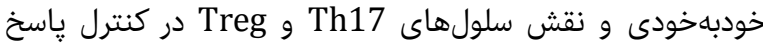

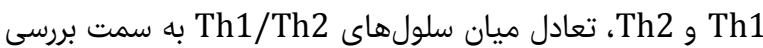

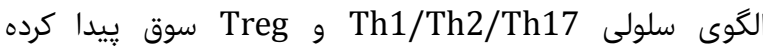
است الستون]

سايتوكاينها سايتوكاينها نقش اساسى در همه مراحل باردارى ايفا مىكنند.

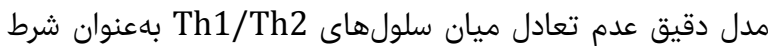

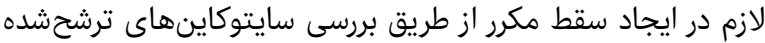

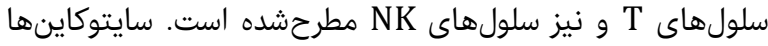

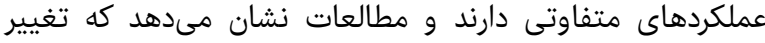

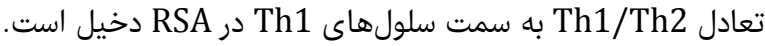

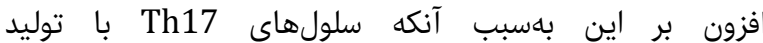
سايتوكاينهايى نظير IL-17 در القاى التهاب و و رد الوكرافت بائ

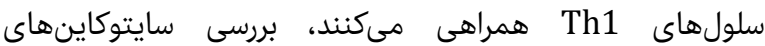
ترشحشده از سلولهاىTh17 نيز اهميت يافته است.

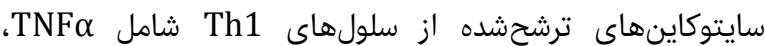

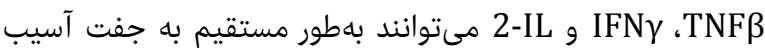

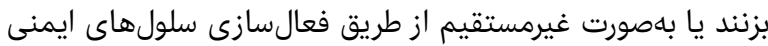

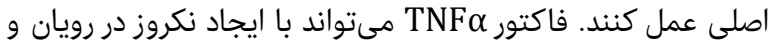

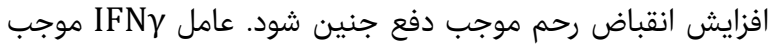

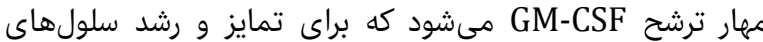

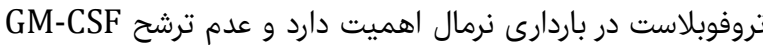

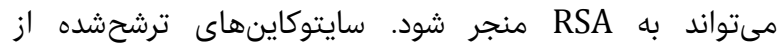

تيروئيديراكسيداز با افزايش تعداد سقط همراه است و تتقريباً در

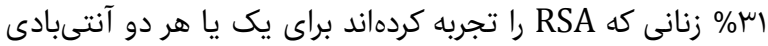

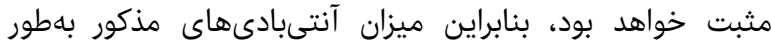

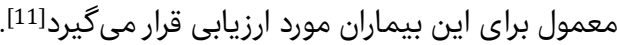

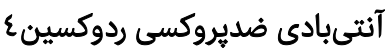

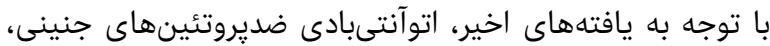

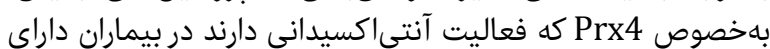
سابقه سقط مكرر خودبه خودى يافت مى شوند.

مكانيزمهاي الوايميون بيانكر باسخ ايمونولوزيك مائيك مادر در براير

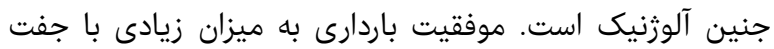

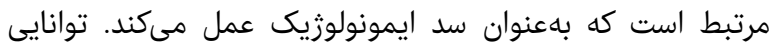

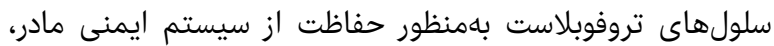

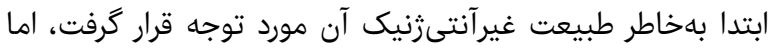

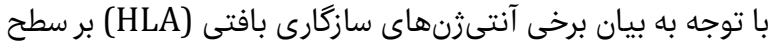

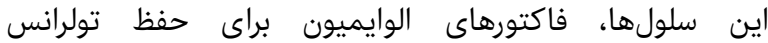
ايمونولوزيك مادر مورد نياز خواهند بود [4] التهائ.

الوآنتىبادىها طى فرآيند باردارى، سيستم ايمنى مادر، HLA يدرى رادي را بهاعنوان

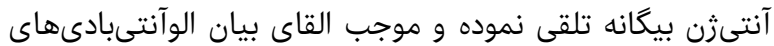

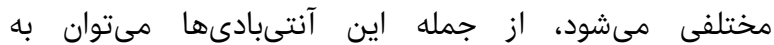

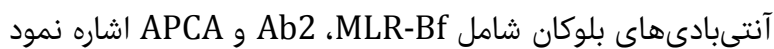

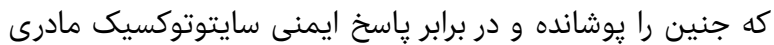

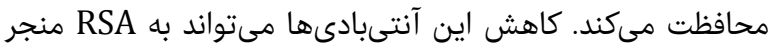

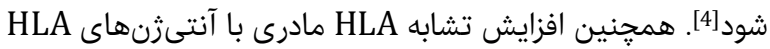

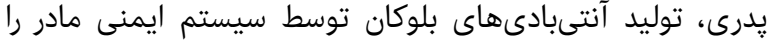

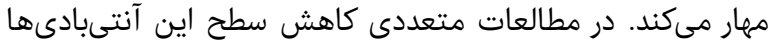

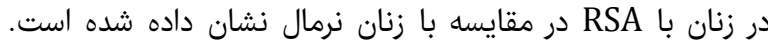

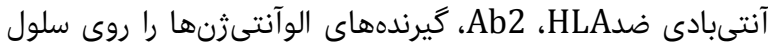

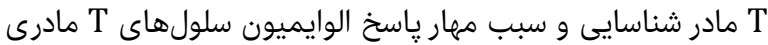

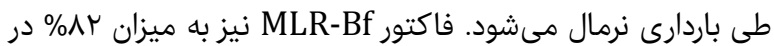

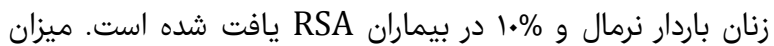

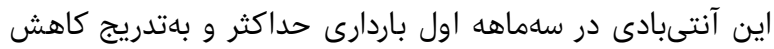

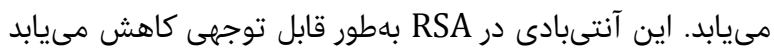

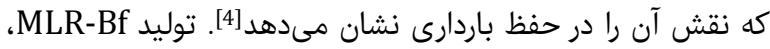

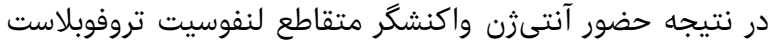
(TLX) آنتى ثنيك مشترك با مولكولهاى

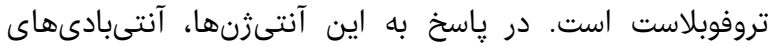

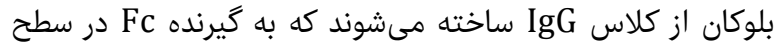

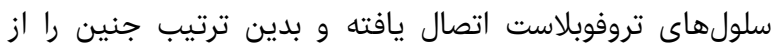

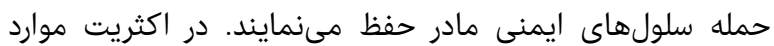

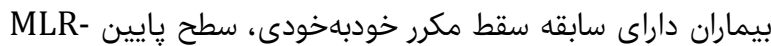

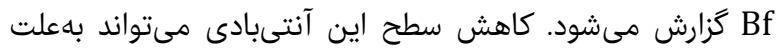

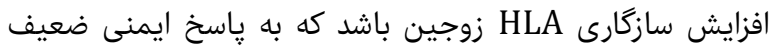

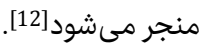

فعاليت سلول

فعاليت، بلوغ و تكثير سلولهاى NK تحت تالثير سايتوكاينهاى

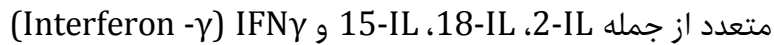
افزايش مىيابد. به اين ترتيب بررسى سلولهاى NK فعالشده در 


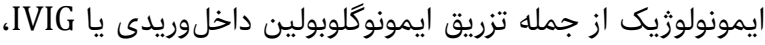
آنتىTNF- $\alpha$ ، G-CSF و لنفوسيت تريترايى اشاره كرد [24].

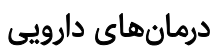

درمان با هيارين از جمله درمانهاي اولئ اوليه براى سقطهاى مكرر بان

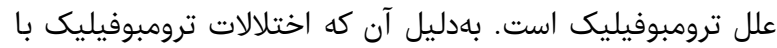

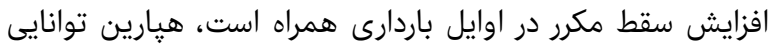

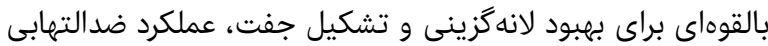

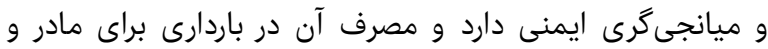

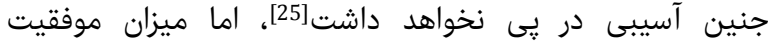

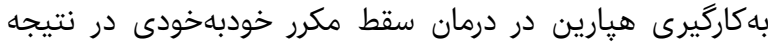

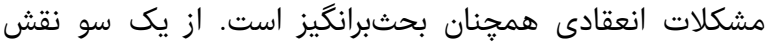

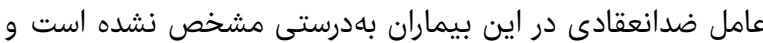

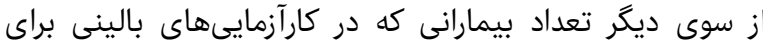

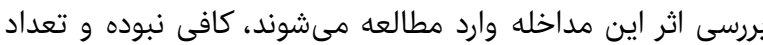

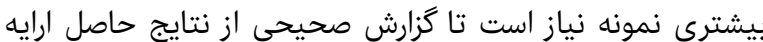

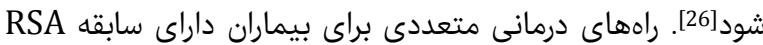

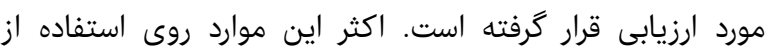

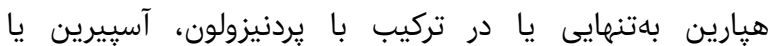
يروزسترون تمركز يافتهاند [27]. تيان.

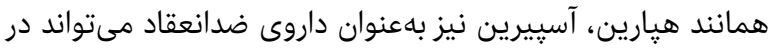

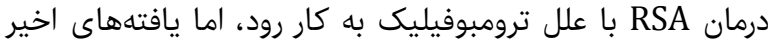

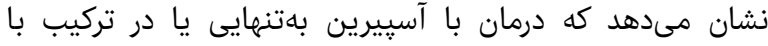

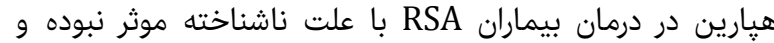

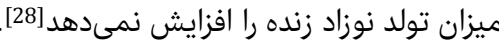

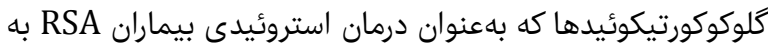

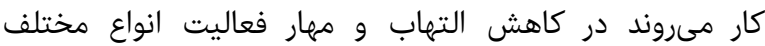

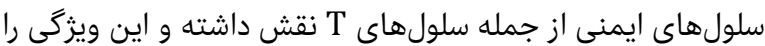

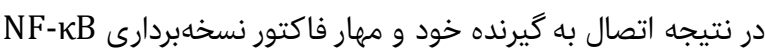

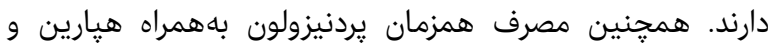
آسييرين با دوز كم در درمان اين بيماران، احتمالاً بهدليل مهار هيرار

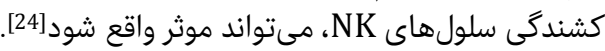

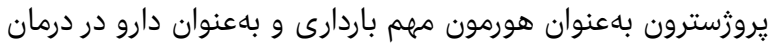

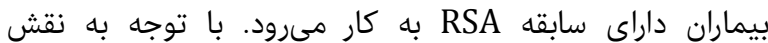

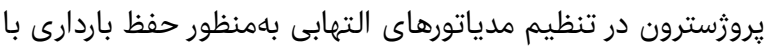

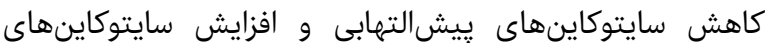

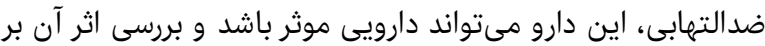

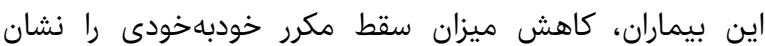

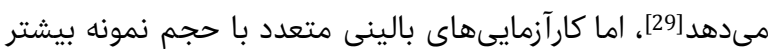
براى تكميل نتايج بهدست آمده، مورد نياز است آتهاي.

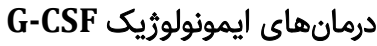
فاكتور G-CSF عضوى از خانواده سايتوكاينى فاكتورهاى محرك

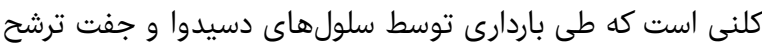

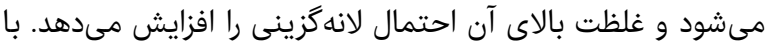

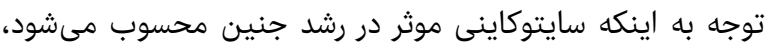

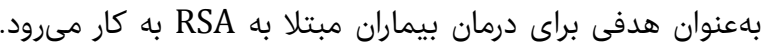

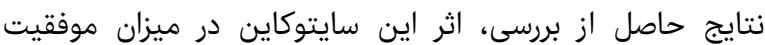

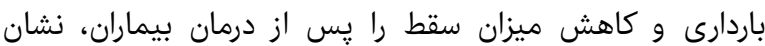

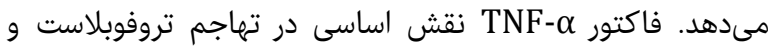

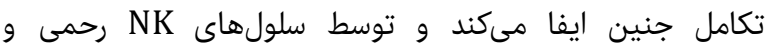
سلولهاى تروفوبلاست توليد و ترشح مىشود [30].

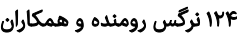

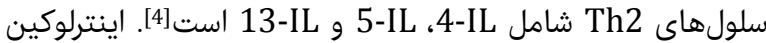

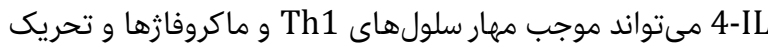

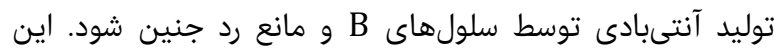

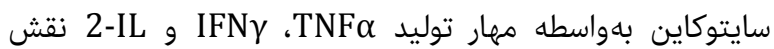

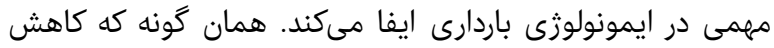

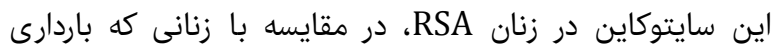

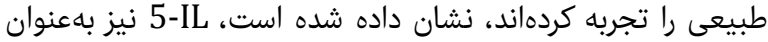

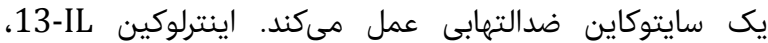

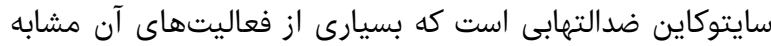

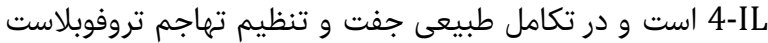

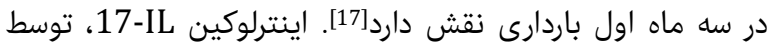

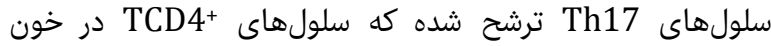

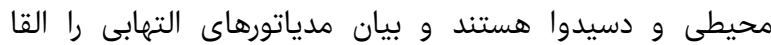

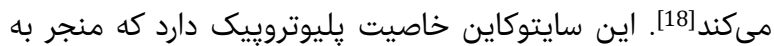

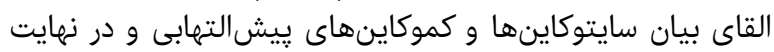

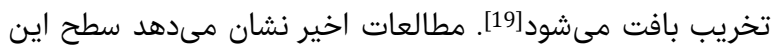

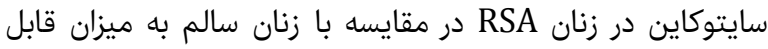

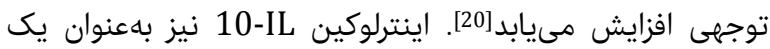

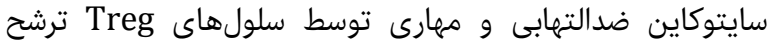

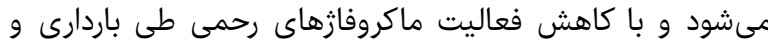

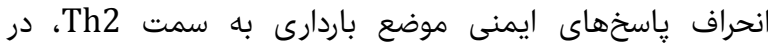

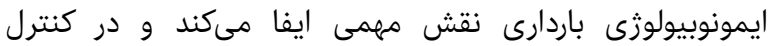

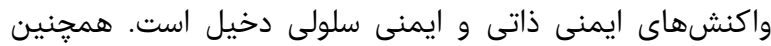

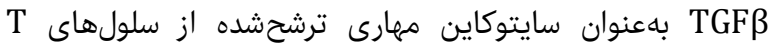

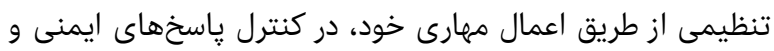

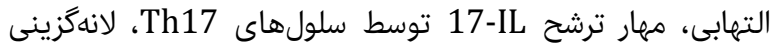
بلاستوسيت، تكامل جفت، كنترل تهاجئ ترابم تروفوبلاست و و تشكيل رويان نقش بسزايى دارد [19)].

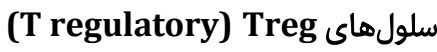

فعاليت سلولهاى T اجرايى نظير Th1، Th2 و Th17 توسط المفاي

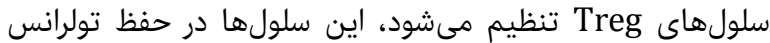

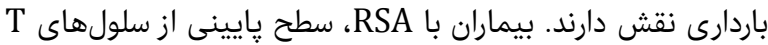

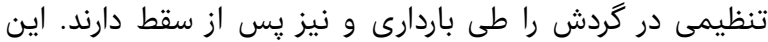

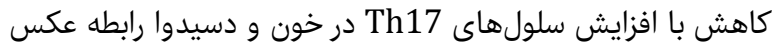

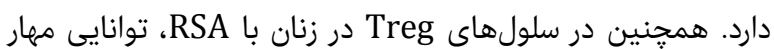

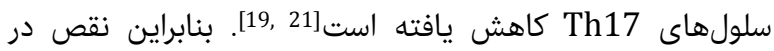

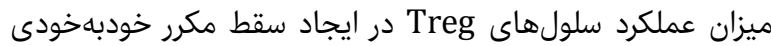

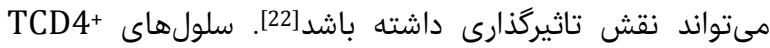

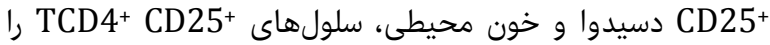

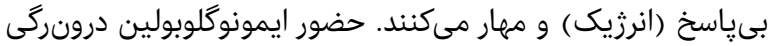

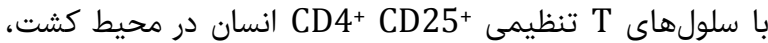

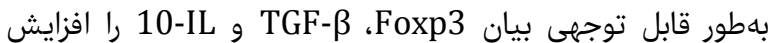

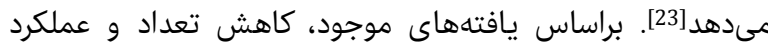
ناقص سلولهاى T تنظيمى در زنان با اين عارضه، شكست بارداري

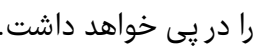

\section{درمانهاى رايج بيماران سقط مكرر خودبهخودى}

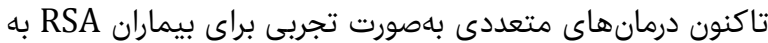

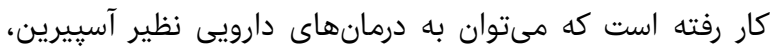

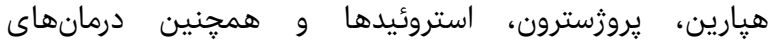


مىشود كه بهطور اختصاصى به TCR اتصال يافته و رياسخ ايمنى إنى

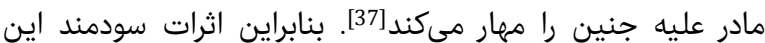

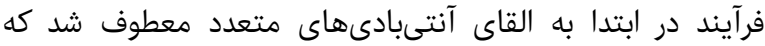

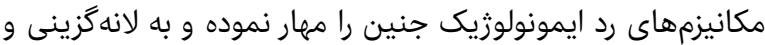
رشد جنين كمك مىكند.

همجنين اثرات مفيد درمان لنفوسيتى شامل مهار آندار اختصاصى و غيراختصاصى سلولهاى T، كاهش سطح گيرنده

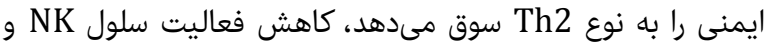

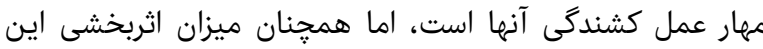

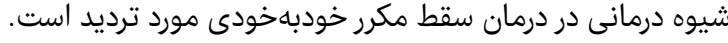

\section{نتيجه گليرى}

با توجه به اين كه علت سقط مكرر خودبهخودى تقريباً در •0\%

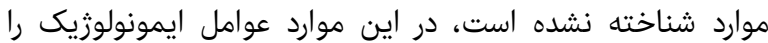

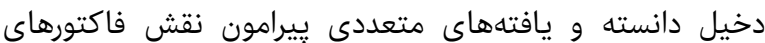

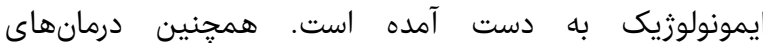

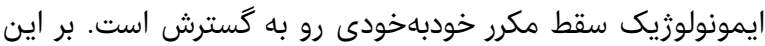

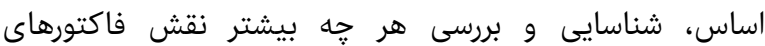

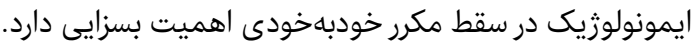

تشكر و قدردانى: موردى از سوى نويسنده كزارش نشده است.

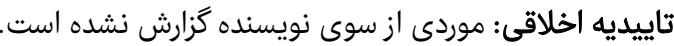

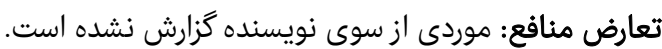

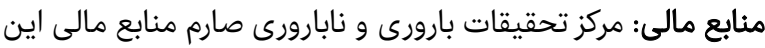

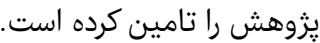

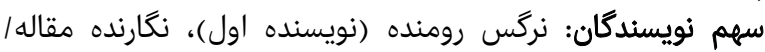

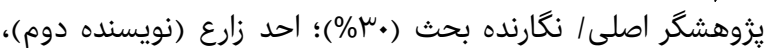

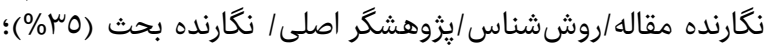

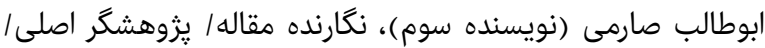

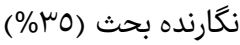

1- Garrido-Gimenez C, Alijotas-Reig J. Recurrent miscarriage: Causes, evaluation and management. Postgrad Med J. 2015;91(1073):151-62.

2- Hogge WA, Byrnes AL, Lanasa MC, Surti U. The clinical use of karyotyping spontaneous abortions. Am J Obstet Gynecol. 2003;189(2):397-400.

3- Christiansen OB, Nielsen HS, Kolte AM. Future directions of failed implantation and recurrent miscarriage research. Reprod Biomed Online. 2006;13(1):71-83.

4- Pandey MK, Rani R, Agrawal S. An update in recurrent spontaneous abortion. Arch Gynecol Obstet. 2005;272(2):95-108.

5- Beaman KD, Ntrivalas E, Mallers TM, Jaiswal MK, Kwak-Kim J, Gilman-Sachs A. Immune etiology of recurrent pregnancy loss and its diagnosis. Am J Reprod Immunol. 2012;67(4):319-25.

6- Hanidziar D, Koulmanda M. Inflammation and the balance of Treg and Th17 cells in transplant rejection and tolerance. Curr Opin Organ Transplant. 2010;15(4):411-5.

7- Sharma S. Natural killer cells and regulatory T cells in early pregnancy loss. Int J Dev Biol. 2014;58(2-4):219-29. 8- Christiansen OB. Reproductive immunology. Mol Immunol. 2013;55(1):8-15.
ايمونيزاسيون غيرفعال با ايمونوكلوبولين داخلوريدى (IVIG)

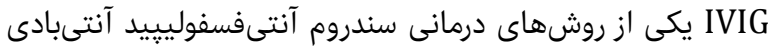

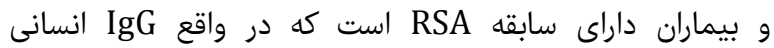

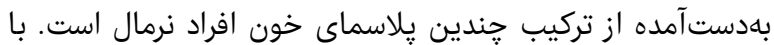

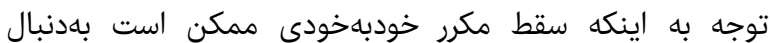

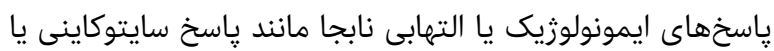

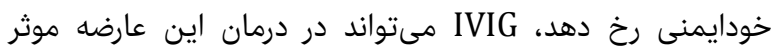

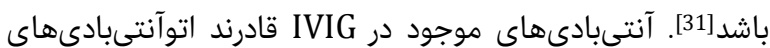

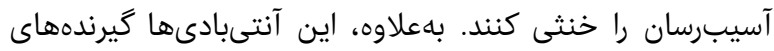

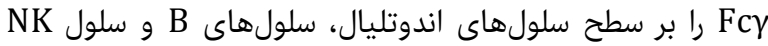

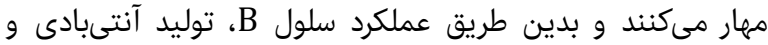

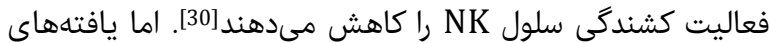

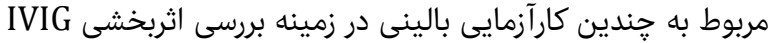

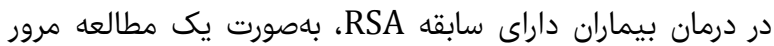

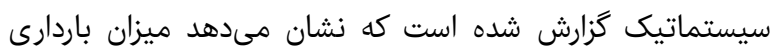

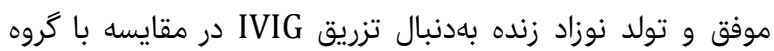
كنترل "كه دارونما دريافت كردهاند" افزايش قابل توني توجهى نداشته

است] (32]

لنفوسيت النرايى

ايمونيزاسيون توسط لكوسيتهاى يدرى (PLI) يا درمان

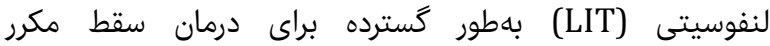

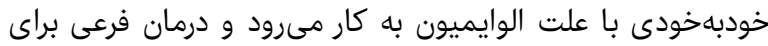

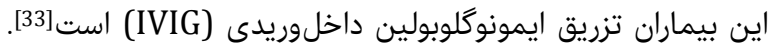

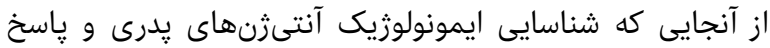

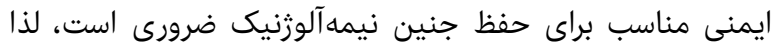

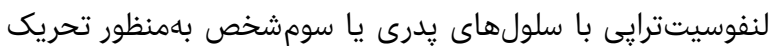

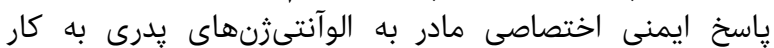

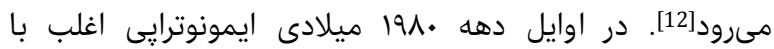

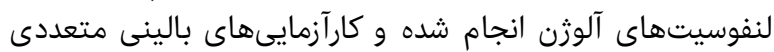

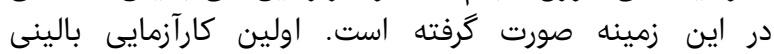

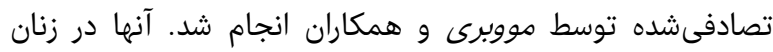

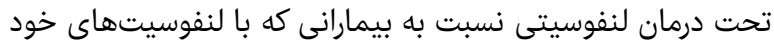
ايمونيزه شده بودند به موفقيت بالاترى دست يافيت بافتند [34].

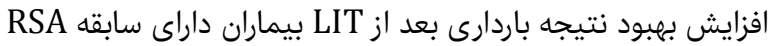

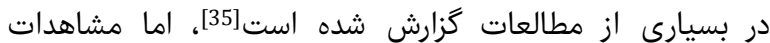

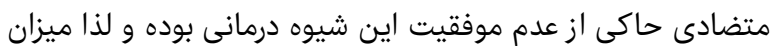

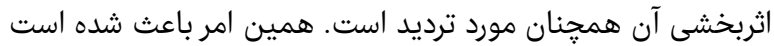
اداره غذا و داروى آمريكا (FDA)، با انجام اين درمان تردين آندان در اين كشور

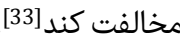
مكانيزمهاى دقيق لنفوسيتترإيى هنوز بهطور كامل شناسايى نشده

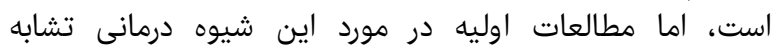

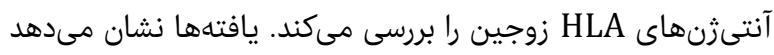

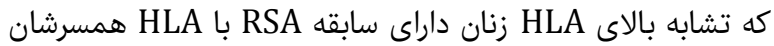

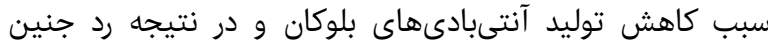

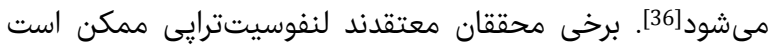

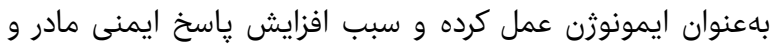

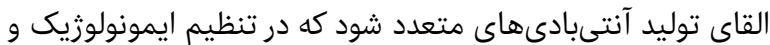

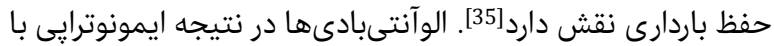

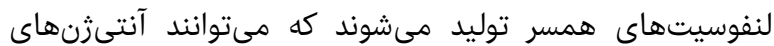

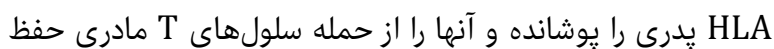

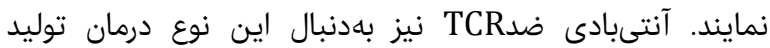


the effect on peripheral NK cells and clinical outcome in patients with unexplained recurrent miscarriage. A double-blind placebo randomized controlled trial. Arch Gynecol Obstet. 2014; 290(4): 757-62.

25- Areia AL, Fonseca E, Areia M, Moura P. Lowmolecular-weight heparin plus aspirin versus aspirin alone in pregnant women with hereditary thrombophilia to improve live birth rate: Meta-analysis of randomized controlled trials. Arch Gynecol Obstet. 2016 Jan;293(1):81-6.

26- Yuksel H, Kayatas S, Boza AT, Api M, Ertekin AA, Cam C. Low molecular weight heparin use in unexplained recurrent miscarriage. Pak J Med Sci. 2014; 30(6): 12327.

27- Fawzy M, Shokeir T, El-Tatongy M, Warda O, ElRefaiey AA, Mosbah A. Treatment options and pregnancy outcome in women with idiopathic recurrent miscarriage: A randomized placebo-controlled study. Arch Gynecol Obstet. 2008;278(1):33-8.

28- Kaandorp SP, Goddijn M, van der Post JA, Hutten BA, Verhoeve HR, Hamulyak K, et al. Aspirin plus heparin or aspirin alone in women with recurrent miscarriage. $\mathrm{N}$ Engl J Med. 2010;362(17):1586-96.

29- Hussain M, El-Hakim S, Cahill DJ. Progesterone supplementation in women with otherwise unexplained recurrent miscarriages. J Hum Reprod Sci. 2012;5(3):248-51.

30- Bansal AS, Bajardeen B, Thum MY. The basis and value of currently used immunomodulatory therapies in recurrent miscarriage. J Reprod Immunol. 2012;93(1):41-51.

31- Practice Committee of the American Society for Reproductive Medicine. Intravenous immunoglobulin (IVIG) and recurrent spontaneous pregnancy loss. Fertil Steril. 2004;82 Suppl 1:s199-200.

32- Egerup P, Lindschou J, Gluud C, Christiansen OB. The effects of immunotherapy with intravenous immunoglobulins versus no intervention, placebo, or usual care in patients with recurrent miscarriages: A protocol for a systematic review with meta-analyses, trial sequential analyses, and individual patient data meta-analyses of randomised clinical trials. Syst Rev. 2014;3:89.

33- Takeshita T. Diagnosis and treatment of recurrent miscarriage associated with immunologic disorders: Is paternal lymphocyte immunization a relic of the past?. J Nippon Med Sch. 2004;71(5):308-13.

34- Mowbray JF, Gibbings C, Liddell H, Reginald PW, Underwood JL, Beard RW. Controlled trial of treatment of recurrent spontaneous abortion by immunisation with paternal cells. Lancet. 1985;1(8435):941-3.

35- Pandey MK, Thakur S, Agrawal S. Lymphocyte immunotherapy and its probable mechanism in the maintenance of pregnancy in women with recurrent spontaneous abortion. Arch Gynecol Obstet. 2004;269(3):161-72.

36- Kano T, Mori T, Furudono M, Ishikawa $\mathrm{H}$, Watanabe H, Kikkawa E, et al. Human leukocyte antigen may predict outcome of primary recurrent spontaneous abortion treated with paternal lymphocyte alloimmunization therapy. Am J Reprod Immunol. 2007;58(4):383-7.

37- Ito K, Tanaka T, Tsutsumi N, Obata F, Kashiwagi N. Possible mechanisms of immunotherapy for maintaining pregnancy in recurrent spontaneous aborters: Analysis of anti-idiotypic antibodies directed against autologous T-cell receptors. Hum Reprod. 1999;14(3):650-5.
9- Kuon RJ, Strowitzki T, Sohn C, Daniel Immune profiling in patients with recurrent miscarriage. J Reprod Immunol. 2015;108:136-41.

10- Shoenfeld Y, Carp HJ, Molina V, Blank M, Cervera R, Balasch J, et al. Autoantibodies and prediction of reproductive failure. Am J Reprod Immunol. 2006;56(56):337-44.

11- Cervera R, Balasch J. Autoimmunity and recurrent pregnancy losses. Clin Rev Allergy Immunol. 2010;39(3):148-52.

12- Khonina NA, Broitman EV, Shevela EY, Pasman NM, Chernykh ER. Mixed lymphocyte reaction blocking factors (MLR-Bf) as potential biomarker for indication and efficacy of paternal lymphocyte immunization in recurrent spontaneous abortion. Arch Gynecol Obstet. 2013;288(4):933-7

13- Bansal AS. Joining the immunological dots in recurrent miscarriage. Am J Reprod Immunol. 2010;64(5):307-15.

14- Zhu LY, Chen X, Xu ZZ, Xu L, Mao T, Zhang H. Changes and clinical significance of peripheral blood helper $\mathrm{T}$ lymphocyte and natural killer (NK) cells in unexplained recurrent spontaneous abortion (URSA) patients after abortion and successful pregnancy. Clin Exp Obstet Gynecol. 2015;42(1):62-6.

15- Yuan J, Li J, Huang SY, Sun X. Characterization of the subsets of human NKT-like cells and the expression of Th1/Th2 cytokines in patients with unexplained recurrent spontaneous abortion. J Reprod Immunol. 2015;110:81-8.

16- Li X, Wang B, Li Y, Wang L, Zhao X, Zhou X, et al. The Th1/Th2/Th17/Treg paradigm induced by stachydrine hydrochloride reduces uterine bleeding in RU486induced abortion mice. J Ethnopharmacol. 2013;145(1):241-53.

17- Xu W, Roos A, Schlagwein N, Woltman AM, Daha MR, van Kooten C. IL-10-producing macrophages preferentially clear early apoptotic cells. Blood. 2006;107(12):4930-7.

18- Nakashima A, Shima T, Inada K, Ito M, Saito S. The balance of the immune system between $\mathrm{T}$ cells and NK cells in miscarriage. Am J Reprod Immunol. 2012;67(4):304-10.

19- Wang WJ, Hao CF, Qu QL, Wang X, Qiu LH, Lin QD. The deregulation of regulatory $T$ cells on interleukin-17producing $\mathrm{T}$ helper cells in patients with unexplained early recurrent miscarriage. Hum Reprod. 2010;25(10):2591-6.

20- Sereshki N, Gharagozloo M, Ostadi V, Ghahiri A, Roghaei MA, Mehrabian F, et al. Variations in T-helper 17 and Regulatory $\mathrm{T}$ Cells during The Menstrual Cycle in Peripheral Blood of Women with Recurrent Spontaneous Abortion. Int J Fertil Steril. 2014;8(1):59-66.

21- Arruvito L, Sotelo AI, Billordo A, Fainboim L. A physiological role for inducible FOXP3(+) Treg cells. Lessons from women with reproductive failure. Clin Immunol. 2010;136(3):432-41.

22- Lee SK, Kim JY, Hur SE, Kim CJ, Na BJ, Lee M, et al. An imbalance in interleukin-17-producing $\mathrm{T}$ and Foxp3(+) regulatory $\mathrm{T}$ cells in women with idiopathic recurrent pregnancy loss. Hum Reprod. 2011;26(11):2964-71.

23- Kessel A, Ammuri H, Peri R, Pavlotzky ER, Blank M, Shoenfeld $Y$, et al. Intravenous immunoglobulin therapy affects $\mathrm{T}$ regulatory cells by increasing their suppressive function. J Immunol. 2007;179(8):5571-5.

24- Gomaa MF, Elkholy AG, El-Said MM, Abdel-Salam NE. Combined oral prednisolone and heparin versus heparin: 